\title{
Frontières
}

\section{Quelques avatars de la fonction sexuelle et leurs accointances avec le deuil et la mort}

\section{Denise Badeau}

Volume 16, numéro 2, printemps 2004

Deuil, blessure vive

URI : https://id.erudit.org/iderudit/1074121ar

DOI : https://doi.org/10.7202/1074121ar

Aller au sommaire du numéro

Éditeur(s)

Université du Québec à Montréal

ISSN

1180-3479 (imprimé)

1916-0976 (numérique)

Découvrir la revue

Citer cet article

Badeau, D. (2004). Quelques avatars de la fonction sexuelle et leurs accointances avec le deuil et la mort. Frontières, 16(2), 74-81.

https://doi.org/10.7202/1074121ar
Résumé de l'article

Cet article présente la sexualité au rendez-vous avec le deuil et la mort à l'occasion de différents avatars de la fonction sexuelle : impuissance (dysfonction érectile, éjaculation précoce ou rapide, éjaculation retardée et anéjaculation), frigidité (absence de désir, baisse de désir, anorgasmie coïtale), vaginisme. Ces avatars sont caractérisés par des pertes, des peurs, des simulacres de la mort dans l'une ou l'autre de ses dimensions. 
Résumé

Cet article présente la sexualité au rendez-vous avec le deuil et la mort à l'occasion de différents avatars de la fonction sexuelle : impuissance (dysfonction érectile, éjaculation précoce ou rapide, éjaculation retardée et anéjaculation), frigidité (absence de désir, baisse de désir, anorgasmie coïtale), vaginisme. Ces avatars sont caractérisés par des pertes, des peurs, des simulacres de la mort dans l'une ou l'autre de ses dimensions.

Mots clés : sexualité - deuil - mort.

\section{Abstract}

This article explores an encounter between sexuality on the one hand and grieving and death on the other through different manifestations of sexual functioning: impotence (erectile dysfunction, precocious or rapid ejaculation, delayed ejaculation and anejacualtion), frigidity (absence of desire, decrease of desire, anorgasmia) and vaginism. These manifestations are characterized by loss, fear, and simulacra of death in one or more of its dimensions.

Key words: sexuality-mourning - death.

\section{Quelques avatars de la fonction sexuelle et leurs accointances avec le deu
et la mort}

\author{
Denise Badeau, Ph.D., \\ professeure retraitée associée \\ au Département de sexologie, UQÀM.
}

Les pertes, le deuil et la mort, entendus au sens propre ou au sens figuré, font partie de l'existence humaine depuis la naissance jusqu'à la mort. Nous le savons théoriquement mais le refoulons dans notre inconscient de sorte que lorsque l'événement douloureux survient, ici la dysfonction sexuelle, il nous prend très souvent pour ne pas dire toujours au dépourvu.

Nous tenterons de mettre en relief les liens pouvant exister entre le deuil, la mort et la dysfonction sexuelle même si, pour certains, il peut paraître étonnant de le faire. Lors de la prestation d'un cours de deuxième cycle en études sur la mort intitulé : «Sexualité, Deuil et Mort», nous avons régulièrement demandé aux étudiants de faire l'exercice suivant: d'indiquer sur une page divisée en deux colonnes, en tête de la première colonne "Sexualité » et en tête de l'autre, «Deuil et Mort » et de procéder par la suite à des associations libres, de part et d'autre, d'une colonne à l'autre. Exemple : du côté de la sexualité, on écrit " ouverture » et vis-à-vis, du côté de la mort: «fermeture ", " enfermement», etc. Chacun était invité à donner libre cours (sans auto- censure) à son inspiration. À la fin de l'exercice, en considérant le contenu de chacune des colonnes, il n'était pas rare de réaliser que ce qui se trouvait dans une colonne aurait très bien pu se trouver dans l'autre, et réciproquement, pour toutes sortes de considérations. Oui, sexualité, mort et les deuils qu'elles obligent à faire présentent des accointances certaines. Ce que Bourgeault exprime en d'autres mots :

Le bonheur, pourtant, n'est possible qu'entremêlé avec un malheur en quelque sorte inhérent, ne serait-ce que dans la tristesse de qui sait, dans le moment même de son bonheur, que celui-ci ne sera pas sans fin ; la santé ne peut être vécue sans que la maladie y prenne place; la vie est marquée dès l'origine par la mort à laquelle elle est vouée. Le jeu des rapports entre bonheur et malheur (sexualité et mort) comme entre santé et maladie, celle-ci étant l'envers révélateur de celle-là, n'est pas simple. Il n'y a pas opposition, si ce n'est sur le plan de la logique, entre l'endroit et l'envers des choses, entre bonheur et malheur, santé et maladie; dans la réalité des vies vécues, il y a plutôt nécessaire convivialité (Bourgeault, 2003, p. 110). 
Pour nous, et nous inspirant de Shearer et Pillot (1986), le deuil comprend à la fois la perte ou la modification de la fonction sexuelle, les sentiments éprouvés à la suite de cette perte ou cette modification et les attitudes intérieures et extérieures qui favorisent ou non l'adaptation. Ici, il s'agira de la perte ou de la modification d'une fonction jugée par le commettant satisfaisante jusqu'alors. Le deuil est un processus normal qui nécessite un travail de longue haleine, travail générateur de stress et grand consommateur d'énergie. Le deuil dépasse donc largement la perte de la fonction ou sa modification.

Le deuil normal peut se révéler bénéfique dans la mesure où il peut amener la personne qui le vit et son ou sa partenaire à inventorier d'autres modalités d'expression de leur génitalité et la capacité de le faire dépendra du deuil premier, c'est-à-dire celui que le petit de l'homme est amené à vivre lors de la séparation de la mère, de l'individuation. Ce que la perte représente pour nous et l'investissement que nous avons fait de l'objet perdu sont souvent beaucoup plus importants que la perte ellemême. Le deuil représente l'un de ces événements de vie qui vont mettre à l'épreuve l'individu (ici, bien souvent le couple) dans ses capacités d'adaptation, de ré-organisation et solliciter le sens qu'il donne à sa vie : « [...] il se pourrait aussi, et l'un n'empêche pas l'autre, que ce qui est perdu ne soit pas objet mais sujet, que ce soit l'essence même de notre être, cette maille qui file » et qu'on peut difficilement remonter ou reprendre (Comte-Sponville, 1992, p. 22).

«Le deuil fait toujours penser à ses limites, à sa finitude, à sa mort. Ce qu'au plus profond de lui-même l'homme ne peut accepter puisque son fantasme narcissique de toute-puissance ou d'omnipotence est indéracinable» (Badeau, 1998, p. 37). Le travail du deuil est ce long processus psychique par lequel la réalité l'emporte nous apprenant à continuer à vivre, à jouir, à travailler, à aimer. La guérison comporterait donc trois processus : acceptation cognitive avec vision rationnelle des événements et des causes, acceptation émotionnelle, changement d'identité. Les obstacles à la guérison qui ont été identifiés par différents auteurs se présentent comme suit: impossibilité de donner un sens à la perte, ambivalence, mésestime de soi.

En termes usuels, nous identifions trois phases dans le travail du deuil: le choc, l'état dépressif, la récupération. La première phase du deuil que nous identifions est celle du choc quand survient la dysfonction ou que l'on en est conscient pour la première fois ; choc plus ou moins prononcé selon l'investissement que nous avions fait de la fonction perdue ou modifiée et selon la brutalité de la perte ou de la modification. Ce choc se traduit par le refus, refus du contenu intérieur de la perte, c'est-à-dire de l'affliction et du travail à faire. On assistera, au cours de cette étape au déni de la réalité, au surinvestissement de la fonction en cause, à la régression du fonctionnement psychique, à une espèce d'engourdissement, ou encore à une espèce de rage, le besoin de tester à tout prix cette fonction, l'humiliation, la perte d'estime de soi, l'atteinte à son identité d'homme ou de femme, la modification de l'image de son corps... La phase centrale du travail du deuil est constituée par un authentique état dépressif au cours duquel s'effectue l'épreuve de la réalité : la perte ou la modification de la fonction est là, bien présente et il faut apprendre à vivre avec, à faire avec. C'est le travail de détachement qui est l'essence même du travail de deuil. Et il se termine dans une période de rétablissement où le moi redevient libre. On voit apparaître douleur, tristesse, désespoir, détresse, affliction, culpabilité, colère contre la fonction perdue ou modifiée et la partie du corps où se concrétise cette perte ou cette modification, agressivité envers soi-même, puis idéalisation de la fonction sexuelle antérieure, surinvestissement, désir de retrouver la fonction telle que connue originellement. Il n'y a pas de croissance psychique possible sans ces mouvements successifs de désinvestissement et de réinvestissement. La capacité de faire un deuil est le prototype de tout changement. L'endeuillé prend conscience que la perte ou la modification a vraiment eu lieu, que rien ne sera plus pareil, parce

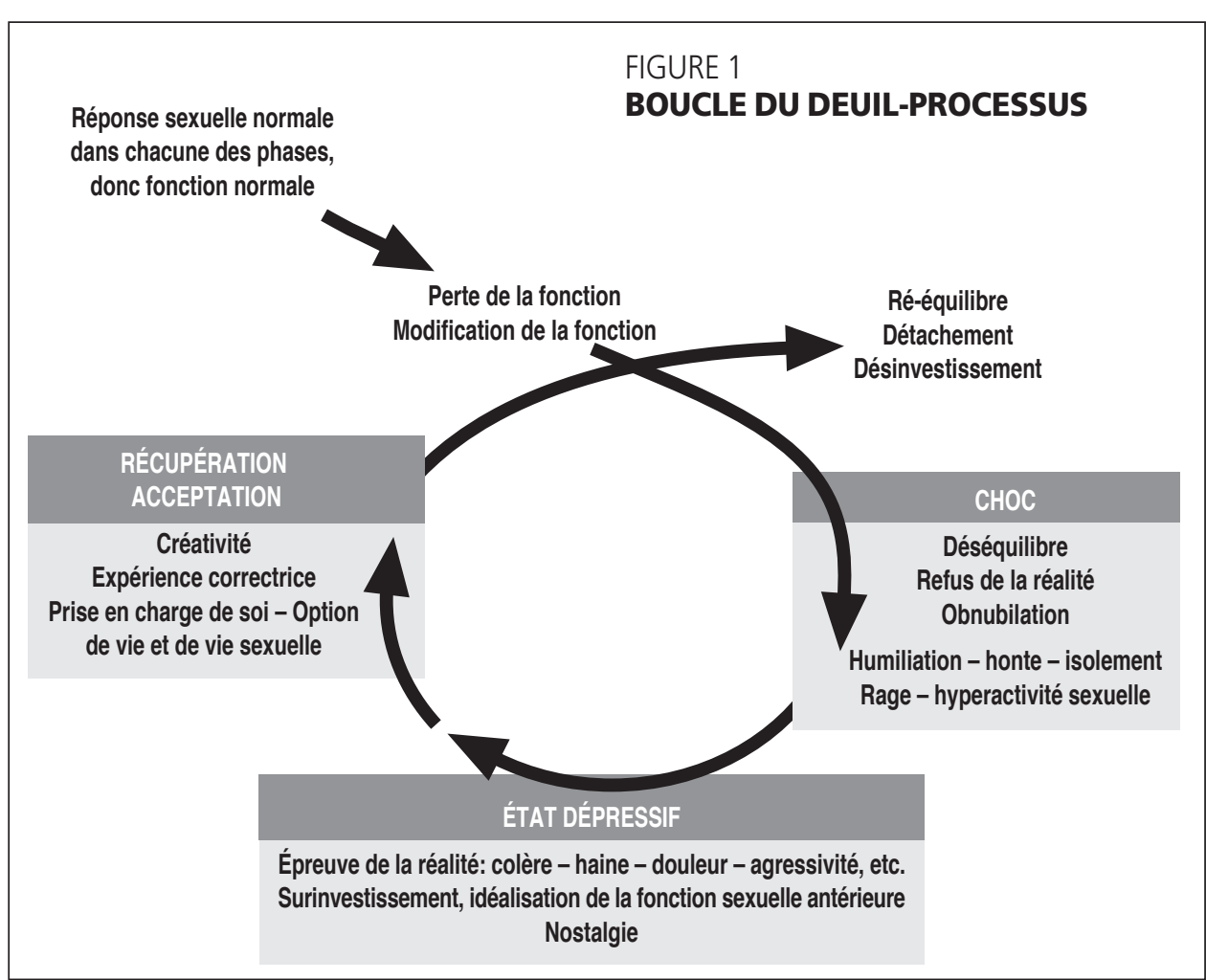

que la perte a engendré l'incertitude, la crainte ou le doute, bien souvent l'humiliation, la dévalorisation. Nous adaptons à notre propos la représentation du deuil de Lamers (1965) dans la figure ${ }^{1}$ ci-dessous.

Selon Hanus (1994), il existe un lien entre le travail du deuil et le travail de création. Voilà qui est intéressant, car la personne vivant une dysfonction sexuelle et sa ou son partenaire auront à explorer d'autres modalités d'expression de la génitalité dont la dysfonction est présente et utiliser cette étape pour le bénéfice de l'individu et du couple. C'est la conversion du trauma en triomphe. Peut-on penser que l'individu qui a réussi le travail du deuil dans ses différentes phases sera plus en mesure d'effectuer une expérience correctrice lorsque survient dans sa vie sexuelle des avatars ou difficultés? «Les mouvements de désinvestissement et de réinvestissement du travail de deuil - déployés dans un temps qui est à la fois celui de l'oubli et du souvenir contribuent dans les meilleurs cas à enrichir le Moi de capacités nouvelles » (Amar et al., 1994).

Considérant la sexualité dans ses différentes dimensions (génitale ou biologique, affective, psychologique, sociale et morale), nous définissons la fonction sexuelle comme étant la réponse sexuelle qui s'élabore en plusieurs phases, dont le nombre et l'appellation peuvent varier selon les auteurs consultés, et est reconnue normale à la suite des stimuli jugés par l'individu comme érotiques. Et les avatars de la fonction seraient les perturbations, les modifications négatives de cette réponse.

\section{FIGURE 1}

ans chacune des phases,

Perte de la fonction Ré-équilibre

Détachement

sinvestissement

RÉCUPÉRATION

CCEPTATION

en charge de soi - Option exuell 
«Kaplan (1979) note ainsi que la réponse sexuelle de tout individu, même en bonne santé physique et mentale, est vulnérable aux situations stressantes auxquelles il est souvent confronté» (Tremblay, 1996, p. 1).

La dysfonction sexuelle serait «un trouble de la réponse sexuelle ou une difficulté importante chez l'homme ou la femme concernant le manque de désir ou l'incapacité de pouvoir répondre adéquatement à l'une ou l'autre des phases de la réponse sexuelle avec une certaine satisfaction» (Germain et Langis, 1990, p. 366).

Plusieurs cliniciens, dont Rosen et Leiblum (1995) notent que la complexité et la chronicité des dysfonctions sexuelles ne font que croître avec le temps et qu'il devient par conséquent plus difficile de les traiter. La raison qu'ils évoquent pour expliquer ce phénomène renvoie à la multiplicité des facteurs liés à l'apparition et au maintien de ces troubles. Mais pour intégrer ces nombreux facteurs qu'ils soient organiques, psychologiques, relationnels ou culturels, et pour tenir compte aussi des interrelations entre ces facteurs, les modèles théoriques actuels se sont complexifiés (Lo Piccolo, 1992, dans Tremblay, 1996, p. 1).

\section{Selon Germain et Langis (1990),}

il n'est toutefois pas toujours facile de déterminer ce qu'est une dysfonction sexuelle ou, inversement, ce qu'est une réponse sexuelle adéquate. Des critères de performance n'existent pas vraiment. Une difficulté ressentie par une personne ou un couple peut être perçue différemment par d'autres. De plus, la satisfaction demeure somme toute très subjective. Pour cette raison, on préfère parler de difficulté et, malgré l'absence d'un seuil bien défini, il est possible de considérer une limite au-delà de laquelle les performances, les fréquences et les capacités sont perçues comme «dysfonctionnelles » (p. 366).

Nous avons choisi de traiter ici des dysfonctions qui par leur appellation contemporaine ou traditionnelle, par la réalité qu'elles engendrent, miment la mort ou entraînent un deuil tel que défini plus haut. Nous nous arrêterons donc aux difficultés érectiles ou impuissance, à l'éjaculation rapide, à l'éjaculation retardée et / ou à l'anéjaculation, à la frigidité (absence de désir, baisse du désir, anorgasmie coïtale), au vaginisme. Ces difficultés traduisent bien souvent des peurs, des anxiétés chez la personne atteinte: anxiété ou angoisse de castration, de séparation ou d'abandon, d'individuation, de ré-engloutissement, de féminitude ou de masculinisation (en ce qui concerne la femme), anxiété de démasculinisation ou de masculinitude (en ce qui concerne l'homme). Elles se produisent souvent quand l'autre sexe ne peut être perçu, être introjecté comme un bon objet ou qu'il est vu comme si différent qu'il suscite de l'envie, de la jalousie, de l'hostilité, de la haine, de la crainte. Ces difficultés sont l'occasion de deuil en ce que l'individu qui les éprouve vit une perte et ne peut se réaliser en tant qu'homme ou femme et en ce que le couple n'a pas accès à l'intimité qu'il souhaiterait.

La perte ou les pertes, telles qu'envisagées dans ce texte, référeront à la perte de fonction ou à des modifications de la réponse sexuelle en regard de l'une ou l'autre phase (désir, excitation, plateau, orgasme, résolution et période réfractaire), à des modifications dans l'expression de la sexualité particulièrement dans sa dimension génitale en raison d'une dysfonction sexuelle secondaire - c'est-à-dire qui apparaît après que l'individu ait connu un fonctionnement normal - ou encore à une dysfonction globale (présente en tout temps) ou partielle (qui apparaît occasionnellement ou à un rythme régulier) (Paradis, Lafond, 1990, p. 168).

La personne (entière, globale) habite sous chacun de ses comportements sexuels. Tout aussi bien sous chacune des dysfonctions érotiques, elle est globale et entière. Ne vouloir que «focuser» la dysfonction érotique peut contribuer à maintenir l'impression de morcellement, justement très souvent reliée à sa dysfonction érotique laquelle existe parce que la personne n'habite plus son érotisme: son érotisme s'est détaché d'elle. La personne est coupée de ses manifestations (Bureau, 1989, p. 6).

La mort, quand elle est évoquée, l'est selon une définition large : mort biologique, clinique bien sûr (personne vivante devenant cadavre, immédiateté, séparation corps-esprit, arrêt des fonctions, rigidité cadavérique, froideur...) mais aussi et peutêtre surtout mort psychologique de l'individu «qui ne sait plus très bien qui il est », c'est-à-dire dont l'identité sexuelle et l'identité tout court sont atteintes, dont l'image corporelle ou l'estime de soi est perturbée, passage de sujet à objet; mort de celui qui n'a plus conscience de vivre "qui ne sait plus qu'il est» (état comateux, confusion, troubles cognitifs de diverses origines) et mort sociale accompagnant la perte ou l'absence de statut (une non-personne) ou la désubjectivation de la personne; on considérera aussi la mort dans les représentations qu'on en a, dans le symbolisme qu'on y associe.

Perte, deuil, mort, sexualité. Des réalités sous-tendues par ces concepts, chacun de nous a une vaste expérience sans toutefois peut-être leur apposer l'étiquette que nous apposons ici sur chacune d'elles.

\section{DYSFONCTION ÉRECTILE (IMPUISSANCE SECONDAIRE TOTALE OU PARTIELLE)}

On entend par difficulté érectile totale ou partielle une «incapacité totale ou partielle à obtenir ou à maintenir une érection suffisante pour permettre le coït, c'est-à-dire la pénétration, les mouvements pelviens et l'éjaculation [...] qu'on désignera parfois sous le nom d'impuissance totale ou partielle» (Blanchette et al., 1996, p. 76). L'homme est déclaré impuissant secondaire quand il échoue dans $25 \%$ de ses tentatives de coït, donc une fois sur quatre. Les causes majeures de l'impuissance seraient une combinaison de facteurs psychologiques, circonstanciels, sociaux, physiologiques ou médicaux, l'éjaculation rapide d'origine psychosociale, l'alcool, l'angoisse de l'échec, une inquiétude au sujet de sa virilité et le fait de douter de son rôle dans la société, une ambivalence au sujet de l'orientation sexuelle, l'absence d'amour ou de stimulation érotique, l'orgueil blessé (femme qui taquine l'homme sur sa capacité sexuelle et/ou sur son apparence physique, qui l'humilie, le ridiculise devant témoin, etc.), la culpabilité, des sentiments d'impuissance, d'inutilité, d'incompétence, l'hostilité à l'égard de la femme à qui il ne veut pas faire le plaisir d'avoir une érection; à qui il ne veut pas faire croire qu'elle est une bonne amante.

Communément étiquettée impuissance, cette dysfonction érotique consiste phénoménologiquement en la perte de pouvoir sur son érotisme, sur son corps, sur son comportement érotique, sur l'érection de son pénis. Cette perte de pouvoir à cause de l'importance et même de la nécessité de l'érection du pénis pour assurer la pénétration vaginale se ressent comme majeure et rejoint même l'identité sexuelle: «Je suis un impuissant».

[...] cette impuissance du pénis l'homme a donc tendance à l'étendre à tout érotisme : il est incapable de toucher, de caresser, de désirer il est incapable d'être viril, d'être un homme. Ensuite, cette contagion s'élargit à d'autres domaines de sa vie: son travail, ses relations sociales, sa compétence à conduire sa vie. [...] 
c'est la tendance de l'impuissance à contaminer tous les domaines de la vie lorsqu'elle rejoint l'identité d'une personne...

(Bureau, 1989, p. 20).

Le privatif «im» dans im-puissance traduit le caractère morbide de cette dysfonction : manque de puissance ou absence de puissance. Ce qui est perdu dans la difficulté érectile, c'est l'objet: virilité, caractéristique privilégiée de l'homme (et de sa partenaire) et valorisée socialement, mais c'est parfois aussi le sujet: «je ne suis plus un homme puisque je ne suis pas capable d'obtenir une érection et d'accomplir avec compétence l'acte sexuel, donc je ne suis plus moi » quand ce n'est pas: «je suis bon à rien maintenant». Perte de l'objet, perte du sujet, perte de soi, perte de la capacité de faire un enfant, c'est-à-dire de donner la vie selon les moyens habituels. De cette perte de puissance sexuelle découleront d'autres pertes de puissance comme l'écrit si bien Jules Bureau (1989) ; cette dysfonction contamine les autres sphères d'activité de l'homme atteint:

L'homme qui a des difficultés érectiles doit défaire la séquence suivante : il est coupable d'envahir le vagin de sa partenaire avec son pénis en érection il laisse à sa compagne le cadre de référence, à elle de décider ce que lui désire - il lui abandonne son pouvoir il n'a plus son pouvoir et il est « impuissant». (Bureau, 1989, p. 28)

Cette perte de puissance entraîne un travail de deuil avec tout ce que cela suppose : choc, état dépressif, adaptation, travail de deuil plus ou moins long, plus ou moins onéreux, plus ou moins douloureux. L'homme atteint risque de disparaître sous l'étiquette «impuissant » qu'il s'appose et qu'odieusement certains professionnels de la santé lui apposent.

La personne vivant un deuil a tendance à se dévaloriser, l'homme vivant une difficulté érectile aussi. La personne vivant un deuil a tendance à idéaliser l'objet perdu, l'homme atteint aussi. L'endeuillé vit du déni, l'impuissant aussi au point qu'il tentera le coït, parfois de façon répétitive et accablante pour la partenaire pour se convaincre que «ce n'est pas vrai », pour conjurer le sort. Cette dysfonction a des accointances avec la mort en ce qu'elle mime certaines caractéristiques de la mort ou du mort (perte de contrôle, impuissance, perte d'identité : passage du statut de sujet à celui d'objet, de personne vivante ayant une identité et un statut à celui d'un amas de cendres) et qu'elle présente aussi parfois des attributs contraires à ceux de la mort (flaccidité / rigidité cadavérique, réversibilité / irréversibilité).

\section{ÉJACULATION RAPIDE, OU PRÉMATURÉE, OU PRÉCOCE, SECONDAIRE}

Comme le relèvent Trudel et al. (1996), les chercheurs ${ }^{2}$ qui ont défini cette dysfonction l'ont fait à partir de différents critères : nombre de poussées pelviennes, durée de la pénétration, fréquence orgasmique de la femme, contrôle sur le réflexe éjaculatoire, capacité d'éjaculer au moment désiré, critères subjectifs du couple (durée du problème, synchronisation de l'éjaculation avec l'orgasme de la femme et le niveau d'insatisfaction vécu dans la dyade). L'éjaculation rapide ou précoce serait «une difficulté à pouvoir ressentir correctement les sensations pré-éjaculatoires, donc à déterminer le point de non retour et à exercer un contrôle volontaire sur le moment de son éjaculation » (Germain et Langis, 1990, p. 374). L'homme atteint éjacule constamment ou fréquemment à la suite d'une stimulation sexuelle minimale ou avant, pendant ou juste après la pénétration et ce, sans qu'il ne le désire et pour de multiples raisons (Trudel et al., 1996, p. 12).

La cause de cette précocité "secondaire» est l'absence de contrôle sur le réflexe éjaculatoire, l'anticipation de l'orgasme, l'incapacité de bien percevoir et de bien situer son niveau d'excitation sexuelle, le désir trop grand de plaire, l'irrégularité des relations, une trop longue période de continence, la distorsion du temps subjectif, l'angoisse de performance, l'anticipation de l'échec, l'anxiété, le stress, le déni des sensations de plaisir, l'ambivalence et l'hostilité envers sa partenaire, l'utilisation d'une forme de contraception inadéquate comme le coït interrompu, le surmenage (Germain et Langis, 1990, p. 375).

\section{L'éjaculation précoce est la}

dysfonction sexuelle masculine la plus répandue. Les études épidémiologiques portant sur la prévalence de cette dysfonction démontrent qu'environ $35 \%$ des hommes éjaculent prématurément. [...] Les troubles émotifs et relationnels associés à l'éjaculation précoce sont souvent importants. En effet, il n'est pas rare que les hommes aux prises avec ce problème se sentent profondément humiliés ou désemparés. (Trudel, 1993, p. 7-8)

On observe que l'homme qui éjacule trop rapidement avec une compagne ressent par ailleurs du pouvoir sur le moment de son éjaculation lorsqu'il se masturbe.

Dans la masturbation, il peut activer ou retarder le rythme de sa caresse et contrôler avec une plus grande précision le déclenchement du réflexe éjaculatoire; il peut aussi augmenter ou diminuer la force érotique des images mentales (les fantaisies) et ainsi s'approcher ou s'éloigner de la période d'irréversibilité du réflexe éjaculatoire. Il en résulte que face à l'éjaculation dans son comportement masturbatoire, il est libre parce qu'il se contrôle il a donc du pouvoir sur lui-même (Bureau, 1989, p. 10).

La présence de l'autre (désirée et/ou excitante, et / ou aimée, spectatrice, évaluatrice, juge peut-être), la montée de son excitation, le pouvoir du mimétisme dans la relation à l'autre, plus particulièrement «dans l'éclatement de l'érotisme », l'ignorance ou l'oubli du dimorphisme sexuel et érotique, la désertion de son corps, le souci de sa performance et du plaisir de sa partenaire semblent cruciaux dans la perte de contrôle de l'éjaculation et

plus il y a de proximité avec l'autre, plus le réflexe éjaculatoire échappe au contrôle. En effet, plus l'identification à l'autre est grande, plus l'amour pour l'autre est présent, plus le désir de l'autre est fort, plus ces proximités engendrent la mise à distance de la pleine conscience masculine et plus le mimétisme primitif conduit la fonction sexuelle: le réflexe éjaculatoire se déclenche (Bureau, 1989, p. 11).

Bien que les femmes, face à cette difficulté de leur partenaire, n'aient pas de réactions similaires et faciles à prévoir, Tordjman (1984) a schématisé ces réactions en cinq phases. Ces phases sont davantage celles que connaît la femme qui est la conjointe permanente: 1) phase d'autoaccusation, 2) phase d'agressivité, 3) phase de résignation, 4) phase d'adultère et 5) phase de rupture. (Germain et Langis, 1990, p. 375)

Ce qui nous amène à aborder la dysfonction inverse du moins dans son appellation, il s'agit de l'éjaculation retardée ou de l'absence d'éjaculation. "Il s'agit d'une inhibition involontaire du réflexe d'éjaculation, le plus souvent accompagnée d'une absence d'orgasme » (Germain et Langis, 1990, p. 376). On pourrait croire que si l'éjaculation rapide est une perte de contrôle du réflexe éjaculatoire, que l'éjaculation retardée ou absente serait attribuable à un trop grand contrôle de ce réflexe. Les spécialistes parleront davantage de résistance que de contrôle, d'isolement plutôt que dans le cas précédent de trop de proximité, de la peur de la différence, de se perdre dans la différence, "d'être phagocyté », de disparaître, du maintien de sa «fragile identité avec rigidité », de peur de l'abandon, peur de salir sa compagne avec son éjaculat, 
d'idéalisation de sa compagne, de refus de se reconnaître en tant qu'homme sexué, sexuel et érotique, de " peur de la paternité », d'expériences de rejet dans le passé avec une ou plusieurs partenaires. [...] Il s'agit d'hommes qui ont des personnalités rigides et compulsives pour qui l'orgasme représente une perte terrible de maîtrise de soi (Marmor, 1981, dans Germain et Langis, 1990, p. 377). L'anéjaculation chez l'homme est très souvent liée à la peur d'engendrer un enfant rival qui entrerait en compétition avec lui et détournerait à son profit une partie de l'amour de sa femme. Elle peut être liée aussi à l'existence de conflits graves, restés muets jusque-là : culpabilité incestueuse refoulée, perception d'une agressivité intense, tendance à l'échec, peur d'une dépersonnalisation (Grafeille, 1984, p. 111).

Léjaculation rapide a des accointances avec la mort en ce que l'homme atteint déserte son corps pour fusionner avec sa partenaire et de ce fait il n'est plus avec luimême, en pleine conscience de ce qu'il vit : ça ressemble à la séparation du corps et de l'esprit dans la mort. La perte de contrôle du réflexe éjaculatoire entraîne nécessairement le deuil de ce contrôle mais aussi du plaisir de vivre une expérience dyadique sexuelle et érotique ensemble, de façon adulte, autonome. L'éjaculation retardée ou l'anéjaculation se caractérise par l'isolement, la peur de la différence, la peur de se perdre dans la différence, la peur de la dépersonnalisation. Dans toutes ces peurs, on rejoint également certains vécus de la mort. L'éjaculation rapide, dans la plupart des cas, entraîne l'insatisfaction de la partenaire, voire sa fermeture à moins qu'en raison de sa propre dysfonction (vaginisme par exemple), de ses propres peurs, la dysfonction de son partenaire l'accommode, mais l'éjaculation retardée particulièrement l'anéjaculation, en raison de la stimulation prolongée qu'elle suppose, peut faciliter la jouissance de la partenaire, mais elle peut aussi la contrarier particulièrement si elle désire faire un enfant et si le sperme par son odeur ou son goût est érotisant pour elle.

\section{LA FRIGIDITÉ}

Symptôme reflet d'un mauvais fonctionnement à un niveau quelconque de l'organisme, la frigidité se traduirait par l'absence d'attrait pour les rapports sexuels. La frigidité dont on parlait dans des temps pas si éloignés de nous recouvre, à notre point de vue, deux entités pathologiques : l'absence de désir et l'absence de plaisir. Nous en traiterons sous le vocable d'absence ou d'inhibition du désir, de baisse du désir et d'anorgasmie coïtale. Même si le terme a été consacré à une dysfonction féminine, on ne peut nier une réalité semblable rencontrée chez l'homme que l'on désignera sous le vocable d'absence ou de baisse de désir et d'anhédonie. Cette condition est issue de différentes causes tant psychologiques que physiques, sociales que psychosomatiques. On reconnaîtra entre autres comme cause psychologique la peur d'être mal jugée par le partenaire, d'être reconnue comme aimant le plaisir, jouisseuse et jouissante, la peur de la grossesse et des responsabilités qu'elle entraîne, le manque d'intimité sexuelle, l'incapacité à vivre l'événement.

\section{ABSENCE DE DÉSIR OU INHIBITION SECONDAIRE DU DÉSIR, BAISSE DU DÉSIR}

Kaplan (1995) définit le désir sexuel comme un besoin pressant ou "une forte envie qui pousse les hommes et les femmes à rechercher, à initier ou à répondre à des situations sexuelles». Selon Trudel et al. (2003), le désir comporte des dimensions biologique (pulsion), psychologique (motivation) et sociale (aspiration). La pulsion pousserait l'individu à rechercher une satisfaction sexuelle. La motivation serait le souhait de se comporter sexuellement. Enfin, l'aspiration référerait à des normes sociales, à des principes, à des attentes qui font qu'une personne juge qu'il est acceptable ou non d'avoir des activités sexuelles.

Précédant et accompagnant l'excitation, le désir est une étape de l'activité sexuelle et est alimenté ou favorisé par la fantasmatique de l'individu, par une valorisation de l'activité sexuelle et par des sentiments amoureux à l'égard du partenaire, autant d'éléments qui peuvent conduire à la poursuite de l'activité sexuelle (Trudel et al., 2003).

L'absence de désir, vue comme une dysfonction secondaire, est souvent liée à une identité faible, à une pauvre estime de soi ( «je ne sais pas qui je suis » ou ce que nous avons défini comme étant la mort psychologique), à une image corporelle négative, à la peur du rejet, à un imaginaire pauvre (aridité mentale), à la pénurie ou à l'absence totale de rêves érotiques, à une expérience antérieure désagréable, traumatisante, douloureuse, à la peur d'être éventrée, déchirée, à l'absence d'attraction ou de capacité d'anticipation, à la routine, à un vide, à une indifférence totale et réelle concernant toute idée de sexe, à la peur de l'intimité, à la peur du plaisir, de l'engagement, à l'autocensure qui peut aller jusqu'à la suppression du désir, à la dépression, au stress. Ajoutons aux facteurs déjà énoncés l'évitement de la sexualité, la passivité, la fréquence des relations sexuelles (trop fréquentes ou rares), l'insatisfaction dans sa relation de couple quand ce n'est pas le désaccord ou les chicanes entre les conjoints, la culpabilité, l'ambivalence des sentiments, le besoin de se punir ou de punir l'autre et le besoin de contrôler, de se contrôler jusqu'à étouffer le désir. Distance, attraction, capacité d'anticipation sont pourtant nécessaires à l'émergence du désir. La baisse du désir sexuel survient après une période d'intérêt sexuel adéquat (Germain et Langis, 1990, p. 370).

Cette diminution semble associée à une détresse psychologique, des événements de vie stressants et des difficultés interpersonnelles. Cette diminution peut être continue ou épisodique en fonction de facteurs psychosociaux ou relationnels. Une problématique se développant autour de l'engagement et de l'intimité peut entraîner une baisse de désir épisodique (Trudel, 2003, p. 13).

La baisse de désir sexuel ou l'absence de désir sexuel a une certaine parenté avec la mort et le deuil en ce sens que le désir sexuel est étroitement liée à la vie, au désir de vivre et de donner ou générer la vie. «Kaplan établit un parallèle entre les troubles du désir et les personnes ayant vécu dans des familles où le plaisir était absent. Plusieurs ont aussi peur de perdre le contrôle dans l'abandon au plaisir...» (Germain et Langis, 1990, p. 371).

D'ailleurs, la dépression caractérisée par une baisse de l'intérêt à vivre, est presque toujours accompagnée d'un désinvestissement érotique et, à la limite, d'une désérotisation de l'existence. Le désir de mourir peut apparaître précisément quand l'érotisation de la vie n'est plus possible. L'érotisation (imaginaire ou réelle) maintient la mort à distance et constitue peut-être la défense la plus efficace contre l'angoisse de la mort. Même dans les conduites sexuelles masochistes et apparemment autodestructrices, le but ultime n'est pas la mort, mais, au contraire, le dépassement de celle-ci à travers l'expérience orgastique (l'orgasme tient lieu alors de victoire sur la mort) (Crépault, 1991, p. 182).

L'anorgasmie coïtale résulte d'une rupture du contact avec soi-même, avec ses goûts et ses conduites particulières. «Selon Kaplan (1974) [...] la dysfonction sexuelle généralisée serait en bonne partie attribuable à l'émergence d'une réponse d'anxiété incompatible avec l'état de relaxation nécessaire à l'apparition des réponses sexuelles » (Trudel, 1988, p. 49). Bureau parlera de

démission de soi-même empêchant une expression libre et harmonieuse de l'être qui reste enfermé. Il ne sert plus

le contact avec l'autre qui devient 
inaccessible. [...] La personne de la femme est évidée et le paraître érotique prend toute la place. De là, la femme devient à ses propres yeux quelque chose «à défaut de se sentir quel-

qu'un ». C'est le passage du statut de sujet à celui d'objet («je suis le vagin à être pénétré»). (Bureau, 1989, p. 37)

L'anorgasmie est donc l'incapacité de parvenir à l'orgasme appelé " petite mort». Parfois la femme résiste à cette "petite mort » par crainte de la " grande mort », par peur de perdre le contrôle; quand elle y cède bien malgré elle, elle devient dépressive, par peur ou manque de confiance en soi et en l'autre, par incapacité de s'abandonner. L'abandon ou le lâcher-prise est pourtant une condition essentielle à la vie, au plaisir, à l'autonomie, à la mort aussi paradoxal que cela puisse paraître.

L'orgasme, comme la mort, se vit dans l'immédiateté (un point « $\mathrm{x}$ » ténu dans le

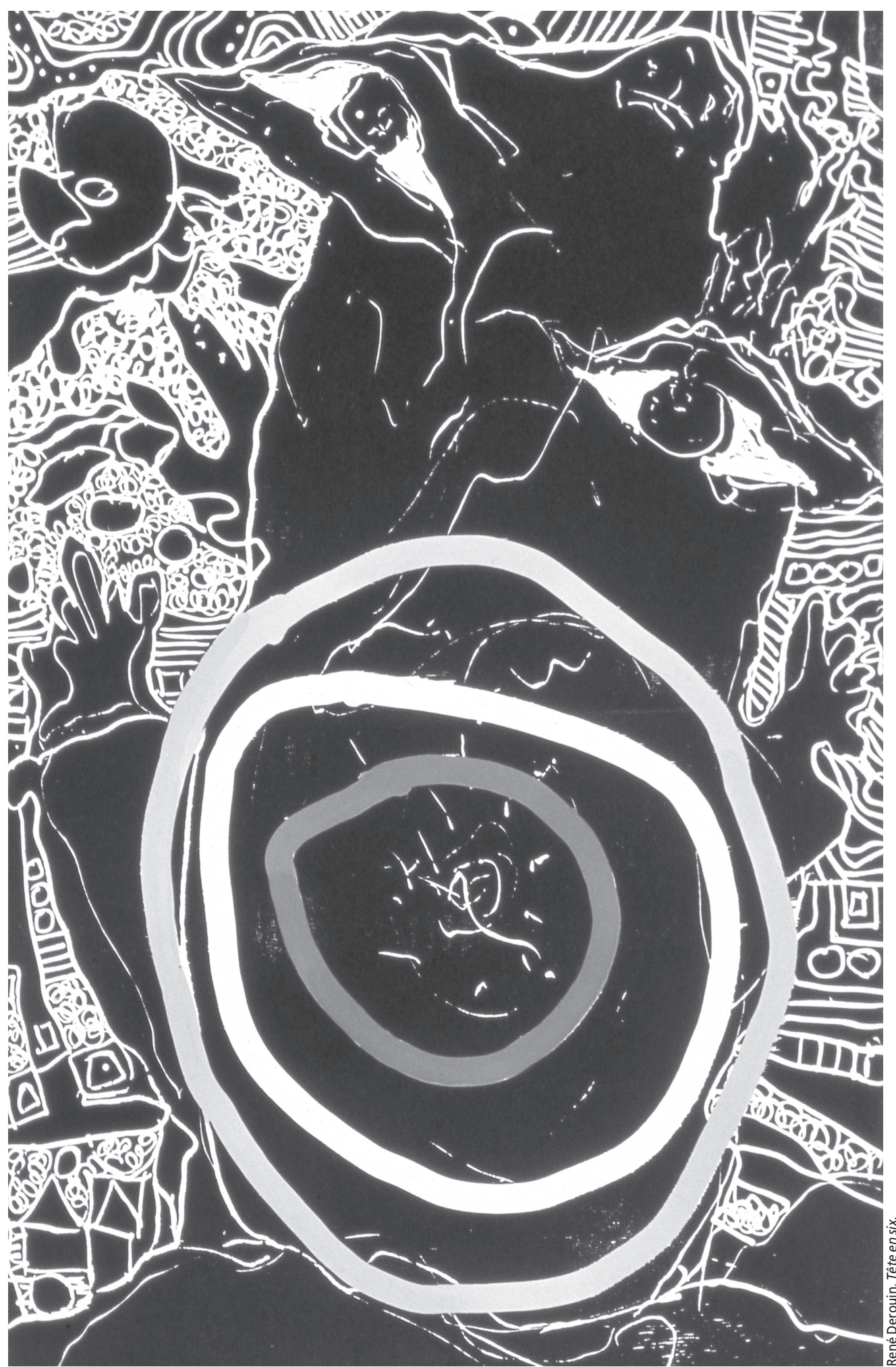

temps), évoque le paroxysme, la démesure, la fin d'un cheminement et l'obligation de passer à l'autre phase de la réponse sexuelle nommée résolution, donc deuil en perspective ; il est l'aboutissement et entraîne parfois une "sensation d'éclatement, d'envahissement (perte ou "éclipse du moi », ceci aussi rappelle la mort, on comprend que la personne atteinte ne se permette pas l'orgasme par crainte de se perdre même si, selon Crépault [1990] il y a également fracture du moi dans l'incapacité d'orgasme), extase (perte de conscience ou état de conscience modifié), rupture avec le réel, flottement d'éternité (Crépault, 1990). Parmi les causes possibles de cette dysfonction, nous reconnaissons l'ignorance de son corps, la peur de la pénétration, de l'envahissement, la frustration associée à l'éjaculation rapide possible du partenaire, le manque de préparation, la peur d'être possédée, le manque d'amour, la routine, l'ennui, etc.

\section{LE VAGINISME VU COMME \\ DYSFONCTION SECONDAIRE}

Le vaginisme, tel que défini dans le DSM-IV (1996), est « une contraction involontaire, répétée, persistante, des muscles périnéaux qui entourent le tiers externe du vagin, en cas de tentative de pénétration par le pénis, le doigt, un tampon ou un spéculum. Chez certaines femmes, la simple anticipation d'une pénétration vaginale peut déclencher un spasme musculaire. La contraction peut aller de légère, responsable d'un certain resserrement et d'une certaine gêne, à sévère, empêchant la pénétration » (p. 602604). Le vaginisme peut être secondaire, généralisé ou situationnel, acquis. La femme peut être capable d'excitation, de lubrification et d'orgasme, mais la pénétration est douloureuse voire impossible (Fliegelman, 1990, p. 15).

Le vaginisme n'est pas un défaut physique, ni une maladie. C'est un état émotionnel dans lequel les causes psychologiques se manifestent par une réponse physique. Il ne s'agit pas tellement d'un problème vis-à-vis de la sexualité sinon de l'intimité d'une femme, de conflit mettant en cause la dépendance et la confiance ainsi que des rapports que cette femme entretient avec son corps. [...] Il s'accompagne d'un sentiment de honte, ce qui explique pourquoi ces femmes et leurs partenaires souffrent en silence et sont réticents à exposer leurs problèmes.

La honte et la gêne que provoquent le vaginisme sont telles que c'est un sujet dont on discute rarement et sur lequel on n'écrit pas (Harrisson, 1996, p. 223). 
Les auteurs qui traitent du vaginisme lui reconnaissent différentes causes organiques ou psychologiques. Parmi les causes organiques les plus fréquemment identifiées dans le vaginisme secondaire, citons une colporraphie extensive antérieure ou colpopérinéorraphie postérieure, une vulvovaginite, une infection du col, de l'utérus ou des annexes, l'herpès génital, des condylomes acuminés, de l'endométriose, des tumeurs cervicales ou des polypes, une lubrification inadéquate en raison d'un manque d'excitation, la constipation, des douleurs pelviennes chroniques de toute étiologie et d'autres douleurs qui nuisent à la relaxation. Parmi les causes psychologiques, nous reconnaissons les suivantes: un traumatisme sexuel comme viol, inceste, molestation, phobie des maladies sexuellement transmissibles et de la grossesse, une homosexualité latente, une dysfonction sexuelle du partenaire: problème érectile ou éjaculation rapide, des problèmes conjugaux non sexuels.

Lintimité affective implique une certaine ouverture à l'autre, un « échange des cœurs » grâce auquel sont partagés les états d'âme, les sentiments les plus profonds, les fragilités émotionnelles. Ce dévoilement, cette mise à nu émotionnelle est possible à la condition d'avoir confiance en l'autre. Dans l'état amoureux, on retrouve une disponibilité quasi totale à l'autre, ce qui est propice à l'intimité amoureuse (Crépault, 1997, p. 81).

Crépault soupçonne chez la femme vaginique l'appréhension d'une "destruction corporelle » et une contamination par le «mauvais pénis». Dans certains cas, ces fantasmes sont si prégnants que la femme ferme son territoire génital et devient vaginique. C'est une fermeture au «mauvais pénis », mais aussi une protection contre «l'homme intrusif et envahisseur» tandis que selon Bureau :

le vaginisme $[\ldots]$ peut résulter $\mathrm{du}$ réflexe de protéger le dernier refuge de la personne. En effet envahie de partout et en tout (dans ses émotions, dans ses valeurs et même jusque dans son identité sexuelle), la femme peut, par réflexe, bloquer l'intromission dans son identité vaginale

(Bureau, 1989, p. 47).

$\mathrm{Au}$ moins en ce lieu, l'envahisseur sera stoppé. Ce dernier n'est pas toujours le partenaire réel, mais peut être l'homme fantasmé - celui qu'elle imagine comme un envahisseur de son intimité.

Le corps passage-au-monde de la personne devient un lieu privilégié pour ériger ses murs et ses défenses. Si l'identité sexuelle se cristallise autour de la fragilité et son paraître dans un esthétisme délicat, toute approche menaçante à cette délicatesse et à cette fragilité suscite un réflexe de fermeture corporelle. Dans ce cadre de « regardez mais ne touchez pas » $[\ldots]$

(Bureau, 1989, p. 47).

Notons que Crépault (1997) et Bureau (1989) quand ils parlent de vaginisme, abordent le thème de fermeture : pour l'un au mauvais pénis, pour l'autre, fermeture corporelle. Le corps de la femme vaginique est une prison bien cadenassée et sous haute surveillance dans laquelle elle s'est emmurée, voire ligotée étroitement de la tête jusqu'aux pieds quand ce n'est pas ensevelie dans un linceul de craintes, de peurs, de fantasmes douloureux. Il n'est pas rare d'observer chez elle des problèmes respiratoires (asthme, grippe, rhume, extinction de voix), des problèmes digestifs (acidité), des problèmes intestinaux (constipation) et parfois de l'anorexie et de l'aménorrhée, des infections multiples. «[...] une boule là, comme un poing au creux du ventre, avec une difficulté à respirer, et toujours l'impression que l'air ne passe pas » (Faye, 1992, p. 26). L'expérience clinique nous a appris que non seulement la femme se ferme corporellement, mais elle s'enferme en outre dans son problème qu'elle perçoit souvent comme une honte, une responsabilité.

Les caractéristiques souvent identifiées chez la femme vaginique sont la peur, les peurs toutes aussi inhibantes les unes que les autres: peur du mauvais pénis qui envahit, qui force les parois du vagin, qui défonce; peur de l'homme, peur du différent; peur viscérale du sexe; peur des relations sexuelles impliquant la pénétration vaginale ; peur de la grossesse ou d'une nouvelle grossesse, peur de l'accouchement. Les fantasmes de destruction, d'envahissement, de contrôle sont très présents chez la femme vaginique. "Les rêves des vaginiques sont des rêves de dangers élémentaires cataclysmiques : fournaises, éclatement phobogène après le réveil, et à peine verbalisables»(Dolto, 1983, p. 183). Le thème de la douleur occupe une place importante dans son discours conscient. Elle répétera: "J'ai peur d'avoir mal... Le pénis est trop énorme pour mon vagin, il va me blesser... »C'est encore plus vrai lorsque le vaginisme est secondaire à une dyspareunie. La peur de la souffrance physique peut toutefois masquer d'autres anxiétés plus profondes.

\section{CONSIDÉRATIONS ET RÉFLEXIONS SUR LES AVATARS DE LA FONCTION SEXUELLE PRÉSENTÉS}

La dysfonction érectile, l'éjaculation rapide et l'éjaculation retardée ou l'anéjaculation ont en commun certaines pertes: de puissance, de contrôle, de soi, de contact avec son corps, de la capacité de se reproduire par les voies habituelles, pour ne nommer que celles-là. Elles ont en commun aussi certaines peurs : de l'échec, de disparaître donc de la mort, de la différence, de la paternité, du rejet, et elles s'accompagnent de tout un éventail de sentiments et d'émotions. L'éjaculation rapide et l'éjaculation retardée ou l'anéjaculation se distinguent en ceci que l'éjaculation rapide peut être attribuable à trop de proximité à l'autre, tandis que l'éjaculation retardée témoigne de la distance à l'autre ou de l'isolement par rapport à l'autre. La réaction de la partenaire de l'homme éjaculateur rapide calque le processus du deuil depuis l'autoaccusation jusqu'à une nouvelle option de vie sexuelle et conjugale.

Les avatars de la fonction sexuelle plus typiquement féminins se caractérisent par un excès de contrôle, de contenance, une identité faible, une image corporelle négative ; ils se caractérisent aussi par des peurs : du rejet, de l'intimité, de l'engagement, de l'abandon, d'être défoncée, de la mort donc, par des sentiments et des émotions divers tout comme dans le deuil. L'anorgasmie et le vaginisme s'accompagnent d'un manque de contact avec soi, avec ses désirs, de fermeture à l'autre et / ou d'enfermement sur soi comme dans un tombeau.

Tous les avatars masculins ou féminins de la fonction sexuelle ont en commun d'envahir, de contaminer les autres sphères de la vie. Ils présentent une étrange parenté ou proximité avec le deuil en ce sens qu'en raison des nombreuses pertes déjà identifiées, dont la plus importante est certes l'objectivation du sujet; ils obligent à un travail de deuil certain et douloureux. De plus, les étapes du deuil comme les phases de la réponse sexuelle varient en nombre et en nomenclature selon les auteurs qui les présentent; le deuil est terminé quand on a complété le processus, la réponse sexuelle est dite normale quand on est passé par toutes les phases avec satisfaction. Ils ont une proximité ou parenté avec la mort en ce sens qu'ils simulent la mort dans l'une ou l'autre de ses dimensions (clinique, psychologique, sociale) et dans ses représentations et ses caractéristiques (insensibilité dans la frigidité, mutisme, asphyxie, paralysie, enfermement ou momification dans le vaginisme). 


\section{RÉPERCUSSIONS DU TRAVAIL DE DEUIL EN COURS SUR LA SEXUALITÉ}

Si l'on regarde maintenant les choses par l'autre bout de la lorgnette, il y a lieu de se demander comment le deuil dont l'individu est affecté à l'occasion des pertes dans sa vie quotidienne (conjugale, familiale, professionnelle), comment ce deuil va influencer l'expression de sa sexualité ? Question intéressante qui pourrait faire l'objet d'un autre article.

Toute la vie de l'être humain est marquée voire ponctuée par la perte d'objets d'amour, par le passage d'une étape de croissance à l'autre, par l'ensevelissement de rêves longtemps bercés, par les ruptures d'amitiés, de contrats, d'ententes, etc. Dans le meilleur des cas, ces pertes, passages, ruptures permettent à l'individu de croître dans son individualité, dans l'estime qu'il a de lui-même, dans son investissement de la vie et de la sexualité comme expression de la vie. Dans le pire des cas, ils l'amènent à se replier sur lui-même, à s'apitoyer sur son sort, à rechercher sans cesse le sein maternel à lui désormais inaccessible. L'objectif ultime du deuil est la réorganisation, le réinvestissement, la formulation de nouveaux objectifs de vie en tant qu'être individué (Badeau, 1998, p. 40).

\section{Bibliographie}

AMAR, N., C. COUVREUR et M. HANUS (1994). Monographies de la revue française de psychanalyse, Le Deuil, Paris, Presses universitaires de France, mai, Avant-propos.

BADEAU, D. (1998). « Perte, deuil et sexualité, une vision sexoanalytique», Frontières, vol. $11, \mathrm{n}^{\circ} 1$, p. 36-40.
BADEAU, D. (2001). "Le vaginisme: de la fermeture à l'enfermement», dans C. CRÉPAULT et Guy LÉVESQUE, Éros au féminin, au masculin, Sainte-Foy, Presses de l'Université du Québec, p. 105-122.

BLANCHETTE, F. et al. (1996). «Diagnostic différentiel de la dysfonction érectile secondaire par l'utilisation de la symptomatologie sexuelle ", Revue sexologique, vol. $4, \mathrm{n}^{\circ} 2$, p. 75-109.

BOURGEAULT, Guy (2003). «Le risque de vivre", Horizons philosophiques. Au risque du bonheur, $\mathrm{n}^{\circ} 1$, vol. 14, automne, p. 108-117.

BUREAU, J. (1989). Une approche existentielle de la dysfonction sexuelle: le modèle de l'identité sexuelle. Notes de cours inédites, Université du Québec à Montréal, Département de sexologie, $52 \mathrm{p}$.

CRÉPAULT, C., J.J. LÉVY et H. GRATTON (1981). «L'expérience orgastique », Sexologie contemporaine, Sainte-Foy, Presses de l'Université du Québec.

CRÉPAULT, C. (1991). « La non-intégration des érotismes fusionnel et antifusionnel: un désordre sexuel négligé», Contraception, Fertilité, Sexualité, vol. 19, n 2 , p. 181-187.

CRÉPAULT, C. (1997). La sexoanalyse, Paris, Payot et Rivages, p. 84.

COMTE-SPONVILLE, A. (1992). "Vivre, c'est perdre », Autrement : Deuils, p. 14-23.

DOLTO, F. (1983). Sexualité féminine, Paris, Scarabée.

FAYE, P. (1992). "Non-consommation de mariage : intimité impossible? ", Sexologies, vol. $1, \mathrm{n}^{\circ} 5$, p. $25-28$

FLIEGELMAN, E. (1990). "Vaginismus sexual emergencies», Medical Aspects of Human Sexuality, juin, p. 15-19.

GERMAIN, B. et P. LANGIS (1990). La sexualité, regards actuels, Montréal, Études vivantes.

GUELPI, J.D. (coordinateur général) (1996). DSM-IV Manuel diagnostique et statistique des troubles mentaux, Paris, Masson.
HANUS, M. (1994). Les deuils dans la vie, deuils et séparations chez l'adulte et l'enfant, Paris, Maloine.

HARRISSON, M.C. (1996) «Le vaginisme» Contraception, Fertilité, Sexualité, vol. 24, $\mathrm{n}^{\circ}$ 3, p. 223-228.

KAPLAN, H. S. (1979). Disorders of Sexual Desire and Other New Concepts and Techniques in Sex Therapy, New York, Brunner-Mazel.

KAPLAN, H. S. (1995). The Sexual Desire Disorders: Dysfunctional Regulation of Sexual Motivation, New York, BrunnerMazel.

LAMERS, W. (1965). Death, Grief, Mourning, the Funeral and the Child, Presentation given at the 84th Annual Convention of the National Funeral Directors Association, Chicago.

PARADIS, A.-F. et J.S. LAFOND (1990). La réponse sexuelle et ses perturbations, Boucherville, Vermette.

SHEARER, R. et J. PILLOT (1986). « Le deuil», Soins palliatifs terminaux, vol. 36, $\mathrm{n}^{\circ} 9$ (11 février), p. 493-500.

TREMBLAY, S. (1996). "Vers une compréhension multimodale des dysfonctions sexuelles" Éditorial, Revue sexologique, vol. $4, \mathrm{n}^{\circ} 2$, p. 1-6.

TRUDEL, G. (1988). Les dysfonctions sexuelles, évaluation et traitement, Sainte-Foy, Presses de l'Université du Québec.

TRUDEL, G., J.-F. DE CARUFEL, P. FORTIER et al. (1996). "L'éjaculation précoce: une recension des écrits », Revue sexologique, vol. $4, \mathrm{n}^{\circ} 2$, p. $7-34$.

TRUDEL, G. (2003). La baisse du désir sexuel, méthodes d'évaluation et de traitement, Paris, Masson.

\section{Notes}

1. Voir aussi Badeau, 1998, p. 37.

2. Liste des chercheurs mentionnés par Trudel et al. (1996) : Colpi et al., 1986; Fanciullacci et al., 1988; Cooper et Magnus, 1984; Lo Piccolo, 1978; Schover et al., 1982; Masters et Johnson, 1970 ; Kaplan, 1974, 1989; Hastings, 1966a. 